\title{
An Error Equivalent Model of Revolute Joints with Clearances for Antenna Pointing Mechanisms
}

\author{
Quan Liu, Sheng-Nan Lu* (1) and Xi-Lun Ding
}

\begin{abstract}
Joint clearances in antenna pointing mechanisms lead to uncertainty in function deviation. Current studies mainly focus on radial clearance of revolute joints, while axial clearance has rarely been taken into consideration. In fact, owning to errors from machining and assembly, thermal deformation and so forth, practically, axial clearance is inevitable in the joint. In this study, an error equivalent model (EEM) of revolute joints is proposed with considering both radial and axial clearances. Compared to the planar model of revolute joints only considering radial clearance, the journal motion inside the bearing is more abundant and matches the reality better in the EEM. The model is also extended for analyzing the error distribution of a spatial dual-axis ("X-Y"type) antenna pointing mechanism of Spot-beam antennas which especially demand a high pointing accuracy. Three case studies are performed which illustrates the internal relation between radial clearance and axial clearance. It is found that when the axial clearance is big enough, the physical journal can freely realize both translational motion and rotational motion. While if the axial clearance is limited, the motion of the physical journal will be restricted. Analysis results indicate that the consideration of both radial and axial clearances in the revolute joint describes the journal motion inside the bearing more precise. To further validate the proposed model, a model of the EEM is designed and fabricated. Some suggestions on the design of revolute joints are also provided.
\end{abstract}

Keywords: Error modeling, Joint clearances, Antenna pointing mechanism, Radial clearance, Axial clearance

\section{Introduction}

In order to achieve real-time tracking and precise pointing on target satellites, dual-axis antenna pointing mechanisms have been widely applied in communication satellites and data relay satellites for satellite-ground and satellite-satellite communication and data transmission. Pointing accuracy of antenna pointing mechanisms plays an important role in dictating the efficiency of the satellite communication system. Since the pointing accuracy is affected by composite factors including joint clearances, thermal load, etc., achieving a high pointing accuracy is a challenging task especially in space.

Clearances in mechanical joints which inevitably exist in all kinks of machines for instance dual-axis antenna

*Correspondence: Ivshengnan5@gmail.com School of Mechanical Engineering and Automation, Beihang University, Beijing 100191, China pointing mechanisms, on one hand, significantly influence performance of the mechanism [1,2]; on the other hand, they are indispensable to allow relative motion between parts and to enable component assemblage.

A large number of researchers have studied on the subject associated with clearance joints [3-6]. Three main methods dealing with clearance in revolute joints are proposed, the spring-damper approach, the massless link approach and the contact force approach [7]. Wang et al. [8] presented a method to determinate panel adjustment values from far field pattern in order to improve the accuracy of large reflector antenna. You et al. [9] modeled and analyzed satellite antenna systems considering the influences of joint clearances and reflector flexibility. Deducing from the joint clearance of manipulators, Ting et al. [10] proposed a simple approach to identify the largest position and direction errors. Zhang et al. [11] provided a dynamic model with multiple clearances of planetary
Springer Open

(c) The Author(s) 2018. This article is distributed under the terms of the Creative Commons Attribution 4.0 International License (http://creativecommons.org/licenses/by/4.0/), which permits unrestricted use, distribution, and reproduction in any medium, provided you give appropriate credit to the original author(s) and the source, provide a link to the Creative Commons license, and indicate if changes were made. 
gear joint to analyze the vibration characteristics. By using "contact-separation" two-state model, Li et al. [12] established the multibody system dynamic equations of two-dimensional pointing mechanism with clearance. Zhang et al. [13] studied the comparison on kinematics and dynamics between the fully actuated 3-RRR mechanism and the redundantly actuated 4-RRR mechanism with joint clearances. According to the probability theory, Zhu et al. [14] presented the uncertainty analysis of robots with revolute joint clearances, which can be applied in both planar and spatial mechanical systems. Bai et al. [15] established a hybrid contact force model to forecast the dynamic performance of planar mechanical systems with revolute joint clearances. Venanzi and Parenti-Castelli [16] proposed a method to evaluate the influence of clearances on accuracy of mechanisms, which works for both planar and spatial mechanisms. Within the framework of finite element, Bauchau et al. $[17,18]$ presented a method to model planar and spatial joints with clearances. Brutti et al. [19] presented a general computer-aided model of a 3D revolute joint with clearances suitable for implementation in multi body dynamic solvers. Taking both radial and axial clearances into consideration, Yan et al. [20] established a synthetic model for 3D revolute joints with clearances in mechanical systems by the contact force approach. Based on the contact force approach, Marques et al. [21, 22] presented a formulation to model spatial revolute joints with radial and axial clearances. In the past decades, modelling of revolute joints with clearances has attracted a wide investigation since it is a significant factor in prediction of kinematic and dynamic performance of mechanical systems. However, most of them only focused on planar revolute joints [23-29], which means only radial clearance has been considered, axial clearance has been scarcely taken into discussion. In fact, because of errors from machining and assembly, thermal deformation and so forth, axial clearance also occurs inevitably in the joint which could cause out-of-plane motion between the journal and the bearing. Combination of both radial and axial clearances in the revolute joint would make the journal motion inside the bearing more complex and unpredictable.

In this paper, by assuming the radial clearance as a virtual massless link with variable length, an error equivalent model (EEM) of revolute joints with clearances is presented, in which both radial and axial clearances are taken into consideration. Compared to the planar model of revolute joints only with radial clearance, journal motion inside the bearing is more abundant and matches the reality better in this model. Besides, the model is more intuitive and graphic than the 3D contact force model of revolute joints in Refs. [20-22] and it is easier to be applied to analysis of pointing accuracy of the spatial dual-axis pointing mechanism (Additional file 1).

\section{Modeling of Revolute Joints with Clearances}

\subsection{Revolute Joints with Clearances}

In theoretical mechanical systems, it is assumed that the rotational axes of two bodies, which are linked by an ideal revolute joint, are coaxial. In practice, there is an unavoidable clearance between the journal and bearing for allowing rotation. Taking the joint clearance into consideration, a relative motion between the two axes, under the constraint of the bearing boundary, will be generated. Figure 1 depicts a cross-section of a revolute joint with radial clearance and its equivalent model $[30,31]$. In the cross-section, the difference between radii of the bearing and the journal defines the range of radial clearance.

In Figure 1(a), the radial clearance can be described as $k$,

$$
\left\{\begin{array}{l}
0 \leq k \leq k_{\max }, \\
k_{\max }=R_{\mathrm{b}}-R_{\mathrm{j}},
\end{array}\right.
$$

where $R_{\mathrm{b}}$ and $R_{\mathrm{j}}$ are radii of the bearing and the journal, respectively.

Figure 1(b) depicts an equivalent model of the revolute joint with radial clearance, on a cross section, by treating the radial clearance as a virtual massless link with variable length. Relative to the situation of an ideal joint, the joint clearances introduce two extra degrees of freedom in the mechanical system, which can be described by a combination of a translation and a rotation. Movement between the journal and the bearing in the range of the radial clearance can be treated as that of a RPR mechanism. In Figure 1(b), $O$ and $O_{1}$ indicate the center of the

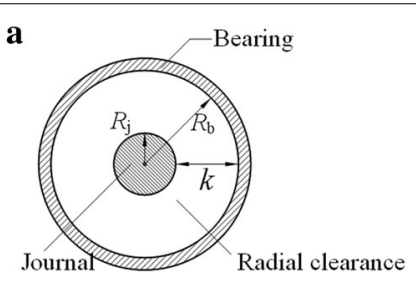

b

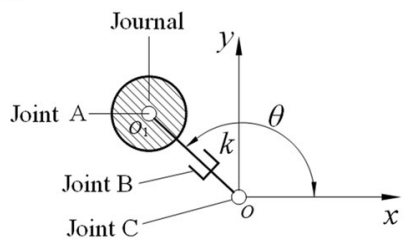

Figure 1 Revolute joints with radial clearance 


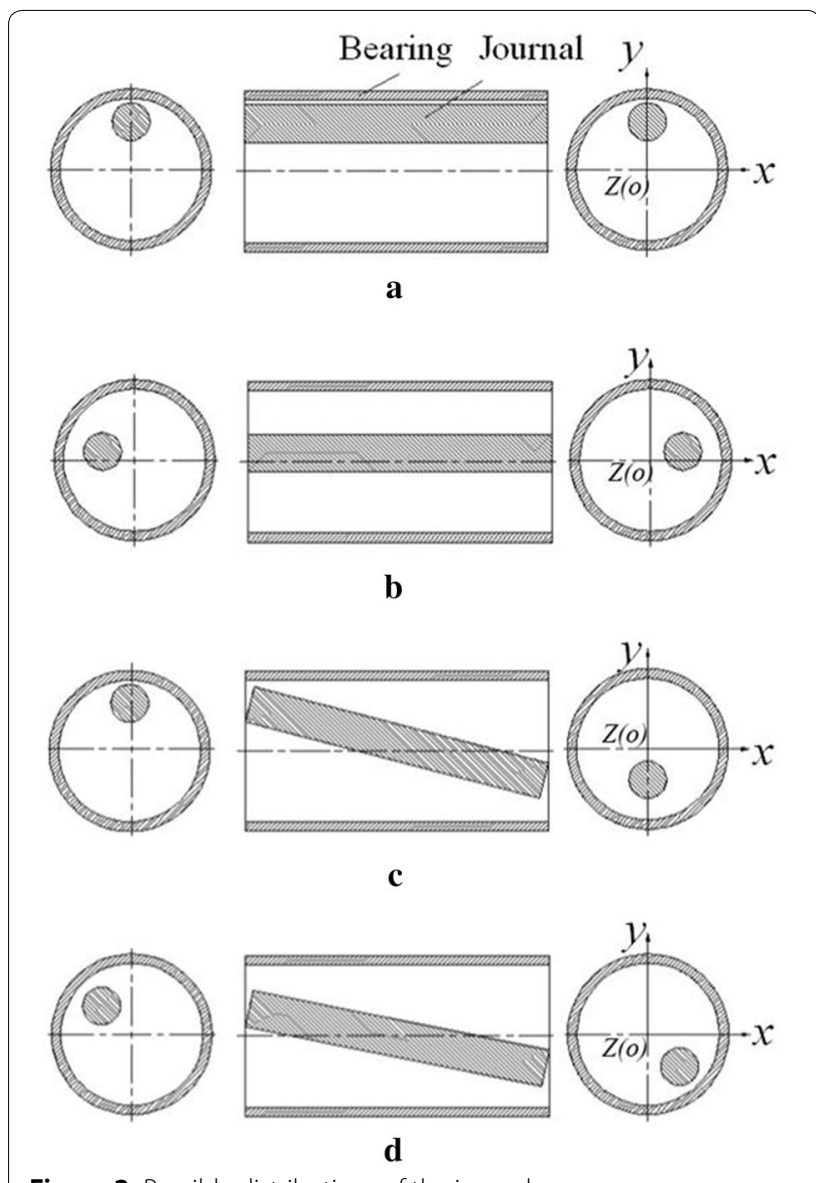

Figure 2 Possible distributions of the journal

bearing and journal, respectively. Joints $\mathrm{A}$ and $\mathrm{C}$ are ideal revolute joints and joint $\mathrm{B}$ is a prismatic joint. Range of motion of the prismatic joint is limited by the maximum radial clearance, that is $\left[0, k_{\max }\right] . \theta$ is the angle from the $x$ axis to the line between the center of bearing and the center of journal, $l_{O O_{1}}, \theta \in[0,2 \pi]$. Therefore, in $O-x y$, coordinate of the journal center is $(k \cos \theta, k \sin \theta)$.

In previous studies, most researchers described the revolute joint with clearances as planar mechanisms. The journal can perform only translational motion with respect to the bearing. However, it is obviously that the assumption does not accord with the physical truth. When joint clearances exist, apart from translation, relative rotation between the bearing and journal can also appear. Therefore, placement of the cross-sections of the journal on two end faces of the bearing can be different which generates different positions and orientations of the journal.

Comparing with the length of a revolute joint, the maximum radial clearance is relatively small. Although, on the end face, projection of cross-section of the journal varies from circle to ellipse along with the rotation between the journal and the bearing, the variation is small enough to be ignored. In the following, we assume that the tiny change does not affect the range of radial clearance, which means that the cross-section of the journal on each end face remains circle. Possible distributions of the journal are shown in Figure 2.

Figure 2(a) describes the journal only performs translational motion under the constraint of the bearing boundary. Figures 2(b) and 2(c) show the journal has both the translational motion and rotational motion while rotational axis of the journal is still on or parallel to the $O-x z$ plane or $O-y z$ plane. General distribution of the journal is illustrated in Figure 2(d).

\subsection{EEM of the Revolute Joint with Clearances}

Known from Section 2.1, physical motion of the journal is relatively complicated. The planar model is inappropriate for obtaining the real error. To more precisely analyze the revolute joint with radial and axial clearances, an EEM of the revolute joint with clearances is established, as shown in Figure 3.

Movement of the journal due to radial and axial clearances is described as that of the platform of a 2RPU-C mechanism. In Figure 3, $O_{1}$ and $O_{2}$, which are the center of the two revolute joints respectively, are fixed. Distance between $O_{1}$ and $O_{2}$ is $l . O_{3}$ and $O_{4}$ are defined as center of the two $\mathrm{U}$ hinges, respectively. $O_{5}$ is the midpoint of segment $l_{\mathrm{O}_{3} \mathrm{O}_{4}}$. The reference plane is parallel to the base plane of the revolute joint and passes $O_{1}$. Plane 1 is parallel to the reference plane and passes $O_{4}$. $O_{3}^{\prime}$ indicates the projection of $\mathrm{O}_{3}$ on Plane 1. $l_{\mathrm{O}_{3}^{\prime} \mathrm{O}_{4}^{\prime}}$ and $l_{\mathrm{O}_{4}^{\prime} \mathrm{O}_{4}}$ are on Plane 1 and perpendicular with each other. Plane 2 is determined by $O_{3}, O_{4}$ and $O_{4}^{\prime}$. It can also be obtained by rotating Plane 1 around $l_{O_{4}^{\prime} O_{4}}$ with an angle $-\alpha$, as shown in Figure 3. $O_{1}-x_{1} y_{1} z_{1}$ is the reference coordinate system. $z_{1}$ axis is along the ideal rotational axis of the revolute joint,

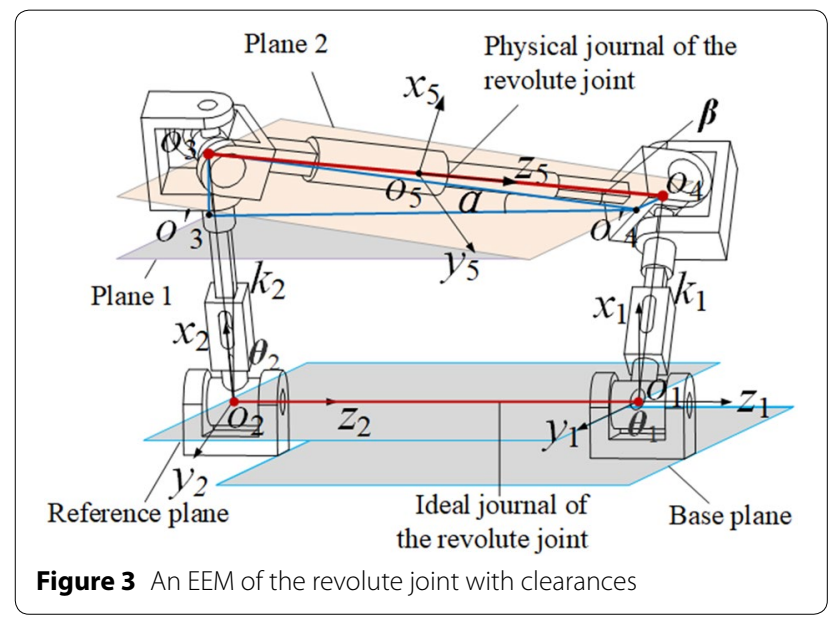


$x_{1}$ axis is perpendicular to the reference plane. The $O_{2}$ $x_{2} y_{2} z_{2}$ coordinate system is obtained by translating the $O_{1}-x_{1} y_{1} z_{1}$ along $z_{1}$ axis with a distance, $-l$, then rotating an angle, $\theta_{2}$, around $z_{1}$ axis. $x_{5}$ axis is perpendicular to Plane 2 and $z_{5}$ axis is along the direction of $l_{\mathrm{O}_{3} \mathrm{O}_{4}}$. In

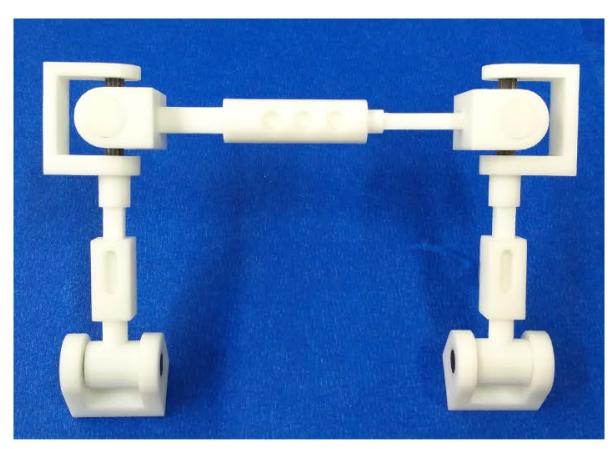

a

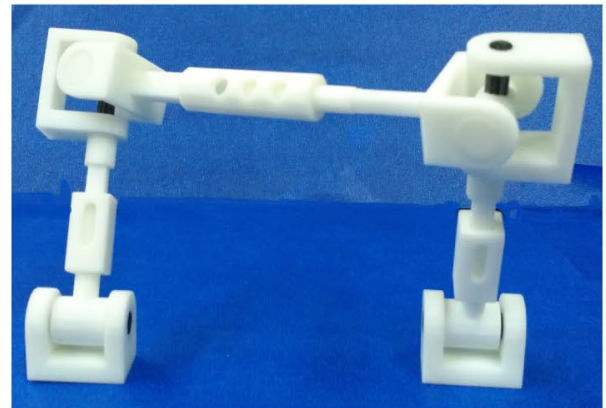

b

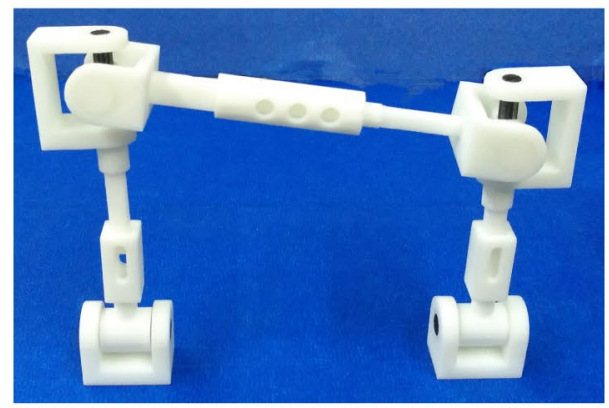

c

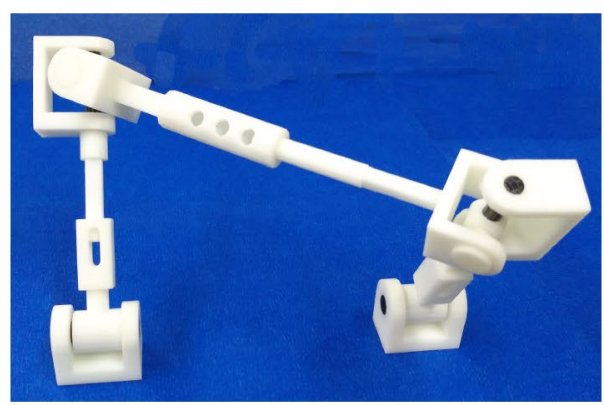

d

Figure 4 Prototype of the EEM
Figure $3, k_{i}(i=1,2)$ is defined as the translational distance of the $i$ th prismatic joint, $k_{i} \in\left[0, k_{i \max }\right] . \theta_{1}$ is the angle from $x_{1}$ axis to the direction of the first prismatic joint, $\theta_{1}, \theta_{2} \in[0,2 \pi]$. In the EEM, the line between $O_{1}$ and $O_{2}\left(z_{1}\right.$ axis) indicates the ideal axis of the revolute joint, while the physical axis is along the line between $\mathrm{O}_{3}$ and $\mathrm{O}_{4}\left(z_{5}\right.$ axis). When $k_{1}=0, k_{2}=0$, the ideal rotational axis and the physical axis are coincident. Known from kinematics of the 2RPU-C mechanism, the physical journal performs both the translational motion and rotational motion. To further validate the proposed model, a model of the EEM is designed and fabricated. Prototype of the EEM is presented as shown in Figure 4.

Configurations of the prototype shown in Figure 4 are corresponding to those in Figure 2. In Figure 4(a), the physical journal has only translational motion. Figures 4(b) and 4(c) show configurations that the journal acts both translational and rotational motion while physical journal of the revolute joint is parallel to the reference plane or the $O-x_{1} z_{1}$ plane constantly. A general configuration of the physical journal is depicted in Figure 4(d). Comparing with the planar clearance model of revolute joints, the EEM of the revolute joint with clearances is more precise since it contains the relative rotation between journal and bearing.

\section{Analysis of the EEM of Revolute Joints with Clearances}

\subsection{Homogeneous Transformation Matrix of the EEM}

Section 2 has presented an EEM of the revolute joint with clearances. As shown in Figure 3, if we define that $O_{i}^{j}=\left[x_{O_{i}}, y_{O_{i}}, z_{O_{i}}\right]^{\mathrm{T}}$ is the coordinate of $O_{i}$ in $O_{j}-x_{j} y_{j} z_{j}$. Direction of the ideal journal is assumed to be $[0,0,1]^{\mathrm{T}}$ in $O_{1}-x_{1} y_{1} z_{1}$. It is obvious that,

$$
\left\{\begin{array}{l}
O_{3}^{2}=\left[k_{2}, 0,0\right]^{\mathrm{T}}, \\
O_{4}^{1}=\left[k_{1} \cos \theta_{1}, k_{1} \sin \theta_{1}, 0\right]^{\mathrm{T}} .
\end{array}\right.
$$

Coordinate of $O_{3}$ in $O_{1}-x_{1} y_{1} z_{1}$ can be calculated as

$$
\left(\begin{array}{c}
O_{3}^{1} \\
1
\end{array}\right)=\left(\begin{array}{cccc}
\cos \theta_{2} & -\sin \theta_{2} & 0 & 0 \\
\sin \theta_{2} & \cos \theta_{2} & 0 & 0 \\
0 & 0 & 1 & -l \\
0 & 0 & 0 & 1
\end{array}\right)\left(\begin{array}{c}
k_{2} \\
0 \\
0 \\
1
\end{array}\right)
$$

Therefore, $O_{3}^{1}=\left[k_{2} \cos \theta_{2}, k_{2} \sin \theta_{2},-l\right]^{\mathrm{T}}$.

In $O_{1}-x_{1} y_{1} z_{1}$, direction of the physical journal, $\boldsymbol{n}$, can be described by the vector from $O_{3}$ to $O_{4}$, which is

$$
\boldsymbol{n}=O_{4}^{1}-O_{3}^{1}=\left(\begin{array}{c}
k_{1} \cos \theta_{1}-k_{2} \cos \theta_{2} \\
k_{1} \sin \theta_{1}-k_{2} \sin \theta_{2} \\
l
\end{array}\right)^{\mathrm{T}} .
$$

As shown in Figure $3, x_{5}$ axis is perpendicular to Plane 2 and $z_{5}$ axis is along the direction of $l_{\mathrm{O}_{3} \mathrm{O}_{4}}$. 
It is known that the vector from $O_{3}$ to $O_{4}$ in $O_{1}-x_{1} y_{1} z_{1}$ is $\boldsymbol{n}$. Similarly, the normal vector of Plane 2 in $O_{1}-x_{1} y_{1} z_{1}$ is

$$
\boldsymbol{p}=(\cos \alpha, 0, \sin \alpha) .
$$

Then direction of $y_{5}$ axis can be calculated as

$$
\boldsymbol{q}=\boldsymbol{n} \times \boldsymbol{p} .
$$

As shown in Figure 3, we can gain the following equations,

$$
\begin{aligned}
& \left\{\begin{array}{l}
O_{3}^{\prime 1}=\left[k_{1} \cos \theta_{1}, k_{2} \sin \theta_{2},-l\right]^{\mathrm{T}}, \\
O_{4}^{\prime}{ }^{1}=\left[k_{1} \cos \theta_{1}, k_{2} \sin \theta_{2}, 0\right]^{\mathrm{T}},
\end{array}\right. \\
& O_{5}^{1}=\left[\begin{array}{c}
\left(k_{1} \cos \theta_{1}+k_{2} \cos \theta_{2}\right) / 2 \\
\left(k_{1} \sin \theta_{1}+k_{2} \sin \theta_{2}\right) / 2 \\
-l / 2
\end{array}\right] .
\end{aligned}
$$

Meanwhile, $O_{5}-x_{5} y_{5} z_{5}$ can be constructed by the following three steps:

Step 1: Translate the $O_{1}-x_{1} y_{1} z_{1}$ coordinate system to $\mathrm{O}_{5}$;

Step 2: Rotate the coordinate system produced in Step 1 with an angle, $-\alpha$, around its $y$ axis;

Step 3: Rotate the coordinate system produced in Step 2 with an angle, $\beta$, around its $x$ axis

Then we can get

$$
{ }_{1}^{5} T=T R_{y}(-\alpha) R_{x}(\beta),
$$

where ${ }_{1}^{5} T$ defines the homogeneous transformation matrix from $O_{5}-x_{5} y_{5} z_{5}$ to $O_{1}-x_{1} y_{1} z_{1}$,

$$
\begin{aligned}
& \tan \alpha=l_{O_{3} O_{3}^{\prime}} / l_{O_{3}^{\prime} O_{4}^{\prime}}=\left(k_{2} \cos \theta_{2}-k_{1} \cos \theta_{1}\right) / l, \\
& \begin{aligned}
\tan \beta & =l_{O_{4} O_{4}^{\prime}} / l_{O_{3} O_{4}^{\prime}} \approx l_{O_{4} O_{4}^{\prime}} / l_{O_{3}^{\prime} O_{4}^{\prime}} \\
& =\left(k_{2} \sin \theta_{2}-k_{1} \sin \theta_{1}\right) / l
\end{aligned} \\
& \alpha=\arctan \left[\left(k_{2} \cos \theta_{2}-k_{1} \cos \theta_{1}\right) / l\right] \\
& \beta=\arctan \left[\left(k_{2} \sin \theta_{2}-k_{1} \sin \theta_{1}\right) / l\right] \\
& R_{y}(-\alpha)=\left(\begin{array}{cccc}
\cos \alpha & 0 & -\sin \alpha & 0 \\
0 & 1 & 0 & 0 \\
\sin \alpha & 0 & \cos \alpha & 0 \\
0 & 0 & 0 & 1
\end{array}\right) \\
& R_{x}(\beta)=\left(\begin{array}{cccc}
1 & 0 & 0 & 0 \\
0 & \cos \beta & -\sin \beta & 0 \\
0 & \sin \beta & \cos \beta & 0 \\
0 & 0 & 0 & 1
\end{array}\right)
\end{aligned}
$$

$$
T=\left(\begin{array}{cccc}
1 & 0 & 0 & \left(k_{1} \cos \theta_{1}+k_{2} \cos \theta_{2}\right) / 2 \\
0 & 1 & 0 & \left(k_{1} \sin \theta_{1}+k_{2} \sin \theta_{2}\right) / 2 \\
0 & 0 & 1 & -l / 2 \\
0 & 0 & 0 & 1
\end{array}\right) .
$$

Therefore,

$$
{ }_{1}^{5} T=\left(\begin{array}{llll}
\mathrm{c} \alpha & -\mathrm{s} \alpha \mathrm{s} \beta & -\mathrm{s} \alpha \mathrm{c} \beta & a \\
0 & \mathrm{c} \beta & -\mathrm{s} \beta & b \\
\mathrm{~s} \alpha & \mathrm{c} \alpha \mathrm{s} \beta & \mathrm{c} \alpha \mathrm{c} \beta & -l / 2 \\
0 & 0 & 0 & 1
\end{array}\right),
$$

where $a=\left(k_{1} \cos \theta_{1}+k_{2} \cos \theta_{2}\right) / 2, b=\left(k_{1} \sin \theta_{1}+k_{2} \sin \theta_{2}\right)$ $/ 2, \mathrm{c} \alpha=\cos \alpha, \mathrm{s} \alpha=\sin \alpha, \mathrm{c} \beta=\cos \beta, \mathrm{s} \beta=\sin \beta$.

\subsection{Constraint Analysis}

In the EEM, the revolute joint with clearances is equivalent to a 2RPU-C mechanism. The two revolute joints on the base plane are assumed to be fixed. Distance between $O_{3}$ and $O_{4}$ is defined as $l_{O_{3} O_{4}}=l+\Delta l . l$ is the distance between $O_{1}$ and $O_{2}$, as well as the distance between $\mathrm{O}_{3}$ and $O_{4}$ along with $z_{1}$ axis. $\Delta l$ is defined as the comprehensive axial clearance, such as axial clearance of machining and axial thermal deformation, etc. Distance between $\mathrm{O}_{3}$ and $\mathrm{O}_{4}$ can be also calculated as,

$$
l_{O_{3} \mathrm{O}_{4}}^{\prime}=\sqrt{k_{1}^{2}+k_{2}^{2}-2 k_{1} k_{2} \cos \left(\theta_{1}-\theta_{2}\right)+l^{2}} .
$$

When $\theta_{2}=\theta_{1}+\pi$, the distance reaches its maximal value,

$$
l_{\mathrm{O}_{3} \mathrm{O}_{4} \max }^{\prime}=\sqrt{\left(k_{1 \max }+k_{2 \max }\right)^{2}+l^{2}} .
$$

Herein, we define $\Delta l^{\prime}$ as,

$$
\Delta l^{\prime}=l_{O_{3} O_{4} \max }^{\prime}-l .
$$

When $\Delta l \geq \Delta l^{\prime}$, the physical journal can freely carry out the translational and rotational motion constrained within the boundary of bearing. However, if $\Delta l<\Delta l^{\prime}$, movement of the physical journal will be limited.

\section{EEM of the Dual-axis ("X-Y" Type) Antenna Pointing Mechanism with Revolute Joint Clearances}

Normally, the dual axes of the antenna pointing mechanism, such as the "X-Y" type antenna pointing mechanism, are orthogonal to each other. Based on the EEM of the revolute joint discussed in Section 2, an EEM of the "X-Y" type antenna pointing mechanism with revolute joint clearances is proposed in this section.

The EEM of the "X-Y" type antenna pointing mechanism can be treated as a combination of two error equivalent models of single revolute joint linked through a prismatic joint, as shown in Figure 5. The ideal journal of 


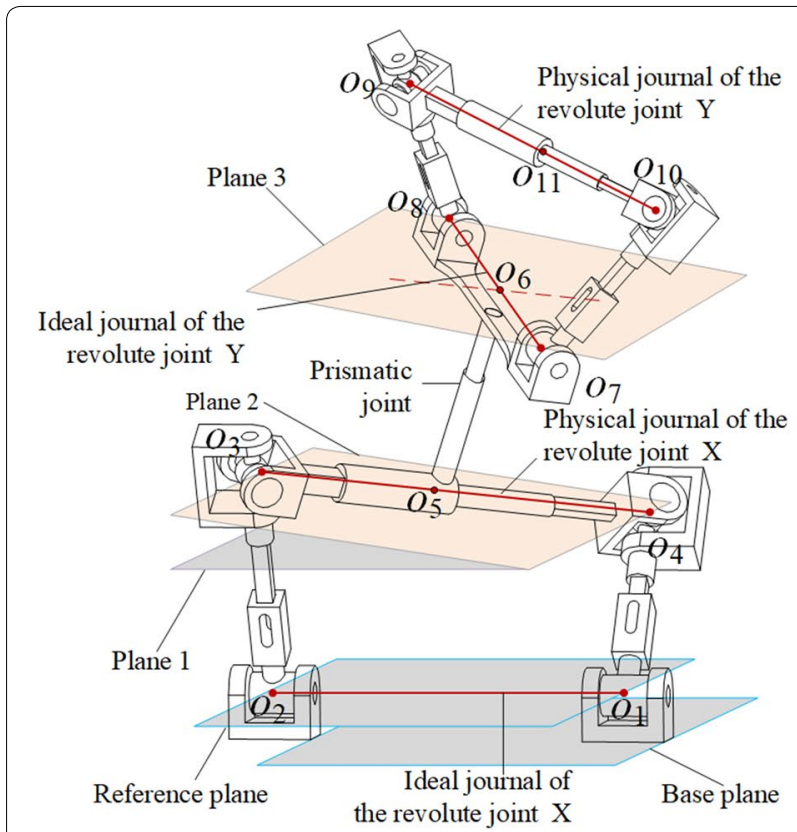

a

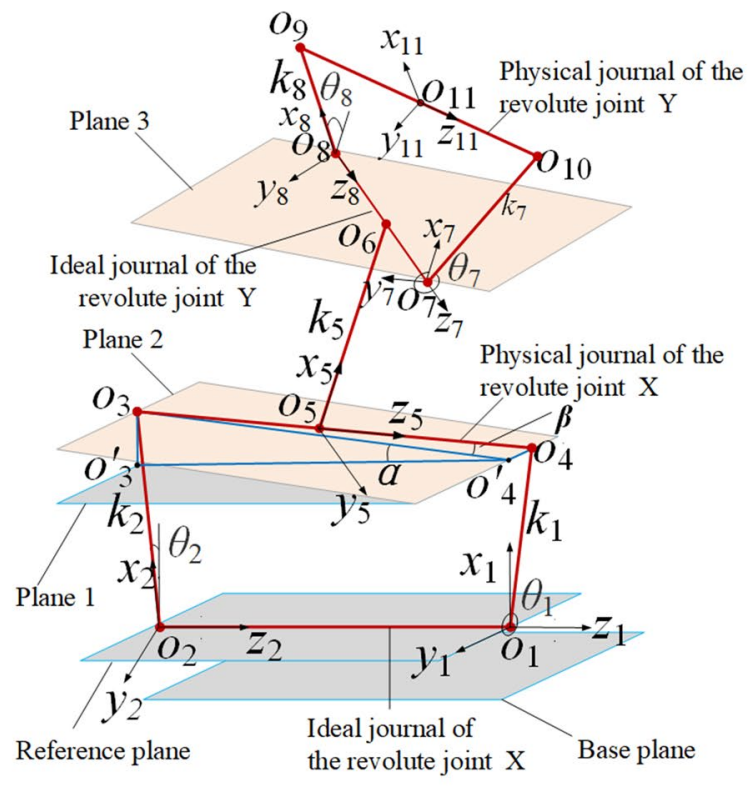

b

Figure 5 An EEM of the " $X-Y$ " type antenna pointing mechanism with revolute joint clearances

the revolute joint $\mathrm{Y}$ is orthogonal to the physical journal of the revolute joint $\mathrm{X}$.

In Figure 5(a), $O_{11}$ is the midpoint of segment $l_{O_{9} O_{10} ;} O_{7}$ and $O_{8}$ express the center of revolute joints in the revolute joint $\mathrm{Y}$, respectively. $\mathrm{O}_{6}$ is the midpoint of segment $l_{O_{7} O_{8}}$. Meanwhile, $O_{9}$ and $O_{10}$ are defined as the center of the $\mathrm{U}$ type hinges in the equivalent model of joint $\mathrm{Y}$.
Plane 3 is parallel to Plane 2, it also passes $\mathrm{O}_{7}$. In addition, $l_{\mathrm{O}_{5} \mathrm{O}_{6}}$ is perpendicular to both Plane 2 and Plane 3. $l_{\mathrm{O}_{7} \mathrm{O}_{8}}$ and $l_{\mathrm{O}_{3} \mathrm{O}_{4}}$ are orthogonal to each other.

Similarly, we can get the homogeneous transformation matrix from $O_{11}-x_{11} y_{11} z_{11}$ to $O_{7}-x_{7} y_{7} z_{7}$,

$$
{ }_{7}^{11} T=\left(\begin{array}{cccc}
\mathrm{c} \omega & -\mathrm{s} \omega \mathrm{s} \tau & -\mathrm{s} \omega \mathrm{c} \tau & m \\
0 & \mathrm{c} \tau & -\mathrm{s} \tau & n \\
\mathrm{~s} \omega & \mathrm{c} \omega \mathrm{s} \tau & \mathrm{c} \omega \mathrm{c} \tau & -l / 2 \\
0 & 0 & 0 & 1
\end{array}\right)
$$

where $\omega=\arctan \left[\left(k_{8} \cos \theta_{8}-k_{7} \cos \theta_{7}\right) / l\right]$, $\tau=\arctan \left[\left(k_{8} \sin \theta_{8}-k_{7} \sin \theta_{7}\right) / l\right]$,

$$
\begin{aligned}
& m=\left(k_{7} \cos \theta_{7}+k_{8} \cos \theta_{8}\right) / 2, \\
& n=\left(k_{7} \sin \theta_{7}+k_{8} \sin \theta_{8}\right) / 2 .
\end{aligned}
$$

The coordinate system $O_{7}-x_{7} y_{7} z_{7}$ can be obtained through the following three steps:

Step 1: Translate $O_{5}-x_{5} y_{5} z_{5}$ with a distance, $k_{5}$, along $x_{5}$ axis.

Step 2: Translate the coordinate system obtained in Step 1 with a distance, $l / 2$, along $y_{5}$ axis.

Step 3: Rotate the coordinate system obtained in Step 2 with an angle, $-\pi / 2$, around its $x$ axis.

Therefore,

$$
O_{7}^{5}=\left[k_{5}, l / 2,0\right]^{\mathrm{T}} .
$$

Homogeneous transformation matrix from $O_{7}-x_{7} y_{7} z_{7}$ to $O_{5}-x_{5} y_{5} z_{5}$ is:

$$
{ }_{5}^{7} T=\left(\begin{array}{cccc}
1 & 0 & 0 & k_{5} \\
0 & 0 & 1 & l / 2 \\
0 & -1 & 0 & 0 \\
0 & 0 & 0 & 1
\end{array}\right)
$$

where $k_{5}$ is the distance between $l_{\mathrm{O}_{7} \mathrm{O}_{8}}$ and $l_{\mathrm{O}_{3} \mathrm{O}_{4}}$. It is mainly affected by the thermal load.

From the above derivation, homogeneous transformation matrix from $O_{11}-x_{11} y_{11} z_{11}$ to $O_{1}-x_{1} y_{1} z_{1}$ can be obtained

$$
{ }_{1}^{11} T={ }_{1}^{5} T_{5}^{7} T_{7}^{11} T \text {. }
$$

\section{Case Studies}

In this section, several case studies are performed based on the proposed EEM. Different values of the geometric parameters are adopted as given in Table 1.

\subsection{Case 1}

In the first case, $\Delta l=0 \mathrm{~mm}$, it means that the revolute joint has only radial clearance and no axial clearance. $\Delta l=0 \mathrm{~mm}$ leads to $k_{1}=k_{2}$ all along. Therefore, the journal can perform only translational motion which 
Table 1 Parameters of the EEM in case studies

\begin{tabular}{llll}
\hline Parameter & Case 1 & Case 2 & Case 3 \\
\hline$\Delta /(\mathrm{mm})$ & 0 & 0.01 & 0.1 \\
$k_{1}(\mathrm{~mm})$ & {$[0,0.05]$} & {$[0,1]$} & {$[0,1]$} \\
$k_{2}(\mathrm{~mm})$ & {$[0,0.05]$} & {$[0,1]$} & {$[0,1]$} \\
$\theta_{1}(\mathrm{rad})$ & {$[0,2 \pi]$} & {$[0,2 \pi]$} & {$[0,2 \pi]$} \\
$\theta_{2}(\mathrm{rad})$ & {$[0,2 \pi]$} & {$[0,2 \pi]$} & {$[0,2 \pi]$} \\
$/(\mathrm{mm})$ & 180 & 180 & 180 \\
\hline
\end{tabular}

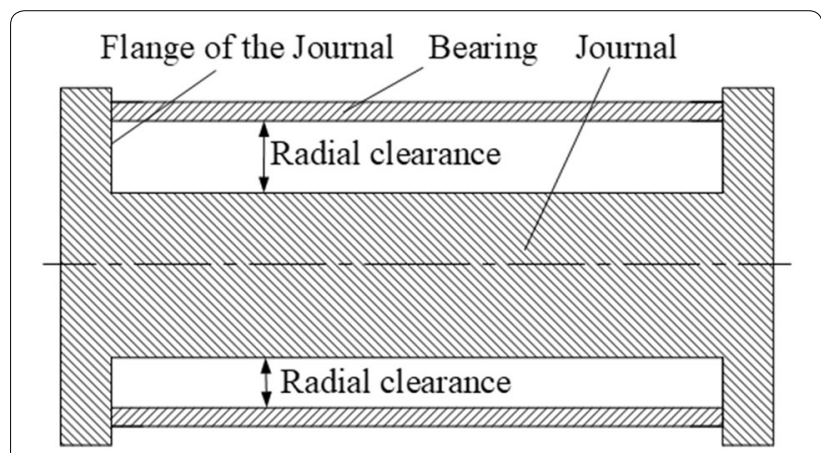

Figure 6 A possible configuration of the journal only with radial clearance

indicates the EEM of the revolute joint with clearances degenerating into the traditional planar model. The ideal journal axis and the physical journal axis are always parallel. Figure 6 depicts the configuration of the journal only have radial clearance.

Since axes of the ideal journal and the physical journal are always parallel to each other, if we define the distance between the two axes is $d$, it is obviously that $d_{\max }=k_{1 \max }=0.05 \mathrm{~mm}$.

\subsection{Case 2}

$\Delta l=0.01 \mathrm{~mm}$ in the second case. From Eqs. (15) and (16), by using the parameters in Table 1, the following can be obtained,

$$
\begin{aligned}
& l_{O_{3} O_{4 \max }}^{\prime}=180.011 \mathrm{~mm}, \\
& \Delta l^{\prime}=l_{O_{3} O_{4 \max }}^{\prime}-l=0.011 \mathrm{~mm} .
\end{aligned}
$$

It is obvious that $\Delta l<\Delta l^{\prime}$, which limits the movement of the physical journal. The radial clearance is restricted by the axial clearance which results in the restrains of $k_{i}$ and $\theta_{i}$. In the 2RPU-C mechanism, the two branches are equivalent. It is allowable to discuss only one of them. Clearances in the RPU branch can be divided into the following two sub-cases.

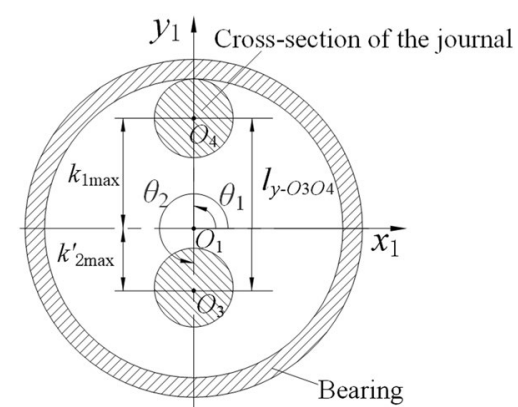

Figure 7 Distribution of the cross-section of the journal in sub-case

Sub-case 1: $k_{1}$ keeps its range from 0 to $1 \mathrm{~mm}$, which allows it to reach its maximum value. $\theta_{1}$ and $\theta_{2}$ still range from 0 to $2 \pi \mathrm{rad}$. We set the values that $k_{1}=k_{1 \max }=1 \mathrm{~mm}$ and $\theta_{1}=\pi / 2 \mathrm{rad}, \theta_{2}=\theta_{1}+\pi=3 \pi / 2 \mathrm{rad}$. The sub-case is shown in Figure 7.

Herein, $l_{y-O_{3} \mathrm{O}_{4}}$ is the distance between $\mathrm{O}_{3}$ and $\mathrm{O}_{4}$ projected on $O_{1}-x_{1} y_{1}$ plane:

$$
\begin{aligned}
& l_{y-O_{3} O_{4}}=\sqrt{(l+\Delta l)^{2}-l^{2}}, \\
& k_{2 \max }^{\prime}=l_{y-O_{3} O_{4}}-k_{1 \max .}
\end{aligned}
$$

Therefore, $l_{y-O_{3} O_{4}}=1.897 \mathrm{~mm}$,

$$
k_{2 \max }^{\prime}=1.897-1=0.897 \mathrm{~mm} \text {. }
$$

It can be seen that the maximum value of $k_{2}$ reduces to $0.897 \mathrm{~mm}$ which indicates the $k_{2}$ can only range from 0 to $0.897 \mathrm{~mm}$.

Sub-case 2: we assume that both $k_{1}$ and $k_{2}$ can reach their maximum values (range from 0 to $1 \mathrm{~mm}$ ). $\theta_{1}$ still ranges from 0 to $2 \pi$, while $\theta_{2}$ is restricted. Assuming that $\theta_{1}=\pi / 2 \mathrm{rad}, k_{1}=k_{2}=1 \mathrm{~mm}$, configuration of the journal in sub-case 2 is shown in Figure 8.

Known from,

$$
\varphi=\arccos \left[\left(k_{1 \max }^{2}+k_{2 \max }^{2}-l_{y-O_{3} O_{4}}^{2}\right) / 2 k_{1 \max } k_{2 \max }\right] .
$$

It can be calculated that $\varphi=0.795 \mathrm{rad}$, which means the angle between the line of the two prismatic pairs can only vary from 0 to $0.795 \mathrm{rad}$.

In case 2 , if the inclination angle of the physical journal is defined as $\psi$, we can get

$$
\begin{aligned}
& \psi_{\max }=\arctan l_{y-O_{3} O_{4}} / l=0.604^{\circ}, \\
& d_{\max }=k_{1 \max }=1 \mathrm{~mm} .
\end{aligned}
$$




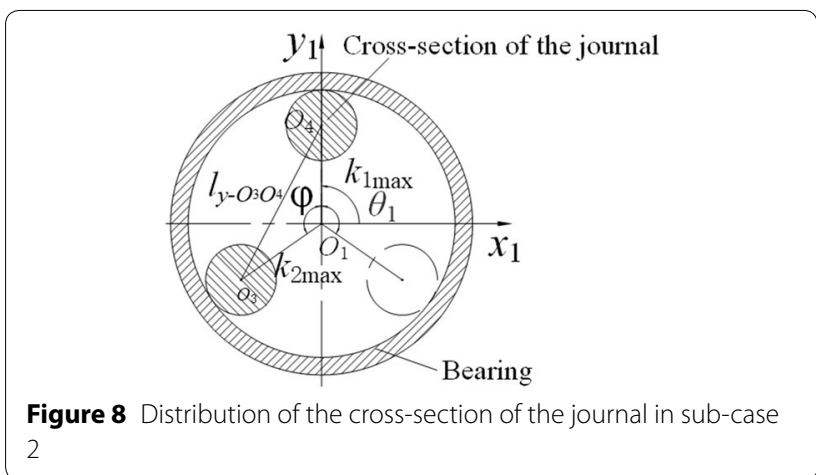

For easier understanding, a possible configuration of the journal in case 2 is described in Figure 9.

\subsection{Case 3}

In the third case, we have $\Delta l \geq \Delta l^{\prime}$, which means the physical journal can freely realize both translational motion and rotational motion constrained within the bearing boundary. In general, the axial tolerance is large enough to allow the physical journal freely to perform rotational motion. Therefore, case 3 is more common in the practical engineering. Figure 10 displays a possible extreme position of the journal in case 3 .

The maximum distance and angle in case 3 is calculated as,

$$
\psi_{\max }=\arctan \left[\left(k_{1 \max }+k_{2 \max }\right) / l\right]=0.637^{\circ},
$$

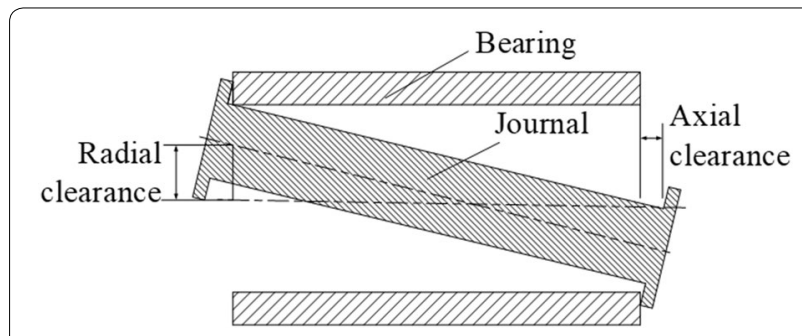

Figure 9 A possible configuration of the journal in case 2

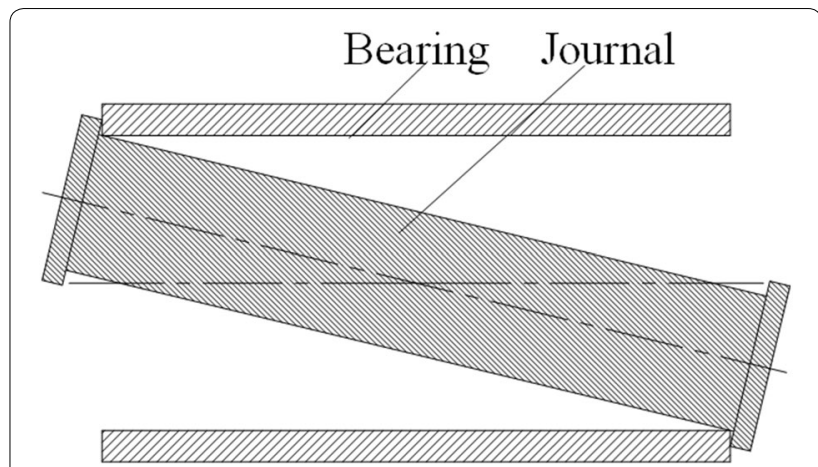

Figure 11 A possible configuration of journal when $\Delta /=\Delta l^{\prime}$

$$
d_{\max }=k_{1 \max }=1 \mathrm{~mm} .
$$

Through the above analysis, it can be known that when $\Delta l=\Delta l^{\prime}$, the physical journal happens to freely realize both translational motion and rotational motion constrained within the bearing boundary. Figure 11 displays a possible extreme position of the journal when $\Delta l=\Delta l^{\prime}$.

\section{Conclusions}

In this paper, an EEM of the revolute joint with clearances is proposed by the virtual bar method. First, the EEM of a single revolute joint with clearances, which is equivalent to a 2RPU-C mechanism, is established. Then, the model is extended for describing the error of a spatial dual-axis ("X-Y" type) antenna pointing mechanism. Comparing to the planar model of the revolute joint only with radial clearance, both radial clearance and axial clearance are involved. Due to the consideration of the rotational motion, the presented model can describe the error of revolute joints with clearances more precisely. It is also revealed that the radial and axial clearances are interrelated and restricted with each other. Three case studies on analyzing the internal relation between the radial and axial clearances are performed which puts forward some suggestions on the design of revolute joints.

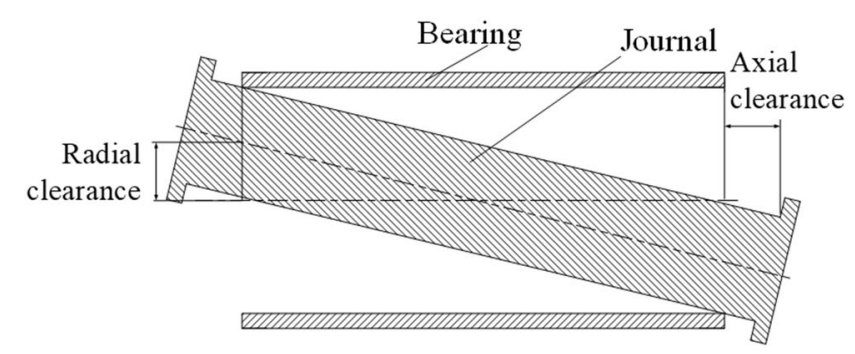

Figure 10 Configuration of the journal in case 3 


\section{Additional File}

Additional file 1. Brief introduction of the paper.

\section{Authors' Contributions}

QL wrote the manuscript with support from S-NL. QL and S-NL developed the theoretical formalism, performed the analytic calculations. QL performed the numerical simulations. X-LD assisted with sample and supervised the project. All authors conceived of the presented idea, discussed the results and contributed to the final manuscript. All authors read and approved the final manuscript.

\section{Authors' Information}

Quan Liu, born in 1992, is currently a postgraduate at Robotics Institute, School of Mechanical Engineering and Automation, Beihang University, China. His main research interests include robotics, pointing mechanism and machine design. E-mail: liuquan2015@buaa.edu.cn.

Sheng-Nan Lu, born in 1987, is currently a postdoctoral researcher at Robotics Institute, School of Mechanical Engineering and Automation, Beihang University, China. Her research interests include kinematic analysis, design of deployable mechanisms, analysis of reconfigurable robots. E-mail: Ivshengnan5@gmail.com.

Xi-Lun Ding, born in 1967, is currently a full professor at Robotics Institute, School of Mechanical Engineering and Automation, Beihang University, China. His main research interests include space mechanisms, kinematics, dynamics, design and control of robotics. E-mail: xlding@buaa.edu.cn.

\section{Competing Interests}

The authors declare that they have no competing interests.

\section{Ethics Approval and Consent to Participate}

Not applicable.

\section{Funding}

Supported by National Natural Science Foundation of China (Grant Nos. 51635002 (Key Program), 51605011, 51275015).

\section{Publisher's Note}

Springer Nature remains neutral with regard to jurisdictional claims in published maps and institutional affiliations.

Received: 29 June 2017 Accepted: 16 April 2018

Published online: 06 May 2018

\section{References}

[1] A Gummer, B Sauer. Modeling planar slider-crank mechanisms with clearance joints in RecurDyn. Multibody System Dynamics, 2014, 31(2): $127-145$

[2] GXWang, H Z Liu. Research progress of joint effects model in multibody system dynamics. Chinese Journal of Theoretical \& Applied Mechanics, 2015, 47(1): 31-50. (in Chinese)

[3] S Dubowsky, F Freudenstein. Dynamic analysis of mechanical systems with clearances-Part 1: formation of dynamic model. Journal of Engineering for Industry, 1971, 93(1): 305.

[4] C M Pereira, A L Ramalho, J A Ambrosia. A critical overview of internal and external cylinder contact force models. Nonlinear Dynamics, 2011, 63(4): 681-697.

[5] P Ravn. A continuous analysis method for planar multibody systems with joint clearance. Multibody System Dynamics, 1998, 2(1): 1-24.

[6] S Erkaya. Trajectory optimization of a walking mechanism having revolute joints with clearance using ANFIS approach. Nonlinear Dynamics, 2013, 71(1-2): 75-91.

[7] P Flores, J Ambrósio. Revolute joints with clearance in multibody systems. Computers \& Structures, 2004, 82(17): 1359-1369.
[8] W Wang, PY Lian, S X Zhang, et al. Effect of facet displacement on radiation field and its application for panel adjustment of large reflector antenna. Chinese Journal of Mechanical Engineering, 2017, 30(3): 578-586.

[9] B D You, H B Zhang, W B Li, et al. Dynamic analysis of satellite antenna system with joint clearance and reflector flexibility. Journal of Aerospace Engineering, 2012, 27(2): 297-307.

[10] K L Ting, J Zhu, D Watkins. The effects of joint clearance on position and orientation deviation of linkages and manipulators. Mechanism \& Machine Theory, 2000, 35(3): 391-401.

[11] H B Zhang, Z K Chen, W Cheng, et al. Vibration characteristics analysis of joint with multiple clearances in space mechanism. Journal of Mechanical Engineering, 2017, 53(11): 44-53. (in Chinese)

[12] S H Li, W B Feng, Q Li, et al. Effect of different gravity environment on the position and collision force of joint with clearance. Journal of Mechanical Engineering, 2015, 51(17): 52-58. (in Chinese)

[13] X C Zhang, X M Zhang. A comparative study of planar 3-RRR and 4-RRR mechanisms with joint clearances. Robotics and Computer Integrated Manufacturing, 2016, 40: 24-33.

[14] J M Zhu, K L Ting. Uncertainty analysis of planar and spatial robots with joint clearances. Mechanism \& Machine Theory, 2000, 35(9): 1239-1256.

[15] Z F Bai, Y Zhao. A hybrid contact force model of revolute joint with clearance for planar mechanical systems. International Journal of Non-Linear Mechanics, 2013, 48(48): 15-36.

[16] S Venanzi, V Parenti-Castelli. A new technique for clearance influence analysis in spatial mechanisms. Journal of Mechanical Design, 2002, 127(3): 446-455.

[17] O A Bauchau, J Rodriguez. Modeling of joints with clearance in flexible multibody systems. International Journal of Solids and Structures, 2002, 39(1): 41-63.

[18] O A Bauchau, C K Ju. Modeling friction phenomena in flexible multibody dynamics. Computer Methods in Applied Mechanics \& Engineering, 2006, 195(50-51): 6909-6924.

[19] C Brutti, G Coglitore, P P Valentini. Modeling 3D revolute joint with clearance and contact stiffness. Nonlinear Dynamics, 2011, 66(4): 531-548.

[20] S Z Yan, W W K Xiang, L Zhang. A comprehensive model for 3D revolute joints with clearances in mechanical systems. Nonlinear Dynamics, 2015, 80(1-2): 309-328.

[21] F Marques, F Isaac, N Dourado, et al. 3D formulation for revolute clearance joints. New Trends in Mechanism and Machine Science, Nantes, France, 2016: 20-23.

[22] F Marques, F Isaac, N Dourado, et al. An enhanced formulation to model spatial revolute joints with radial and axial clearances. Mechanism \& Machine Theory, 2017, 116: 123-144.

[23] J Ma, L F Qian. Modeling and simulation of planar multibody systems considering multiple revolute clearance joints. Nonlinear Dynamics, 2017(90): 1907-1940.

[24] X P Wang, G Liu. Modeling and simulation of revolute joint with clearance in planar multi-body systems. Journal of Mechanical Science \& Technology, 2015, 29(10): 4113-4120.

[25] L X Xu, Y G Li. Modeling of a deep-groove ball bearing with waviness defects in planar multibody system. Multibody System Dynamics, 2015, 33: 229-258.

[26] Z F Bai, Y Q Liu, Y Sun. Investigation on dynamic responses of dual-axis positioning mechanism for satellite antenna considering joint clearance. Journal of Mechanical Science \& Technology, 2015, 29(2): 453-460.

[27] J Ma, L F Qian, G S Chen, et al. Dynamic analysis of mechanical systems with planar revolute joints with clearance. Mechanism \& Machine Theory, 2015, 94: 148-164.

[28] M A B Abdallah, I Khemili, N Aifaoui. Numerical investigation of a flexible slider-crank mechanism with multijoints with clearance. Multibody System Dynamics, 2016, 38(2): 173-199.

[29] Y Y Li, G P Chen, D Y Sun, et al. Dynamic analysis and optimization design of a planar slider-crank mechanism with flexible components and two clearance joints. Mechanism \& Machine Theory, 2016, 99: 37-57.

[30] X Li, X L Ding, G S Chirikjian. Analysis of angular-error uncertainty in planar multiple-loop structures with joint clearances. Mechanism \& Machine Theory, 2015, 91: 69-85.

[31] J L Li, H Z Huang, S Z Yan, et al. Kinematic accuracy and dynamic performance of a simple planar space deployable mechanism with joint clearance considering parameter uncertainty. Acta Astronautica, 2017, 136: 34-45. 